\title{
Coexisting Rathke Cleft Cyst and Pituitary Adenoma Presenting with Pituitary Apoplexy: Report of Two Cases
}

\author{
Florian Gessler, M.D., 1,* Valerie C. Coon, M.D., 1,* Steven S. Chin, M.D., Ph.D., ${ }^{2}$ \\ and William T. Couldwell, M.D., Ph.D. ${ }^{1}$
}

The authors report two cases of coexisting Rathke cleft cyst (RCC) and pituitary macroadenoma. Both patients presented at the university hospital with pituitary apoplexy symptoms of sudden-onset headache while undergoing treatment with Coumadin (warfarin). Magnetic resonance imaging was consistent with a pituitary adenoma in one case and RCC in the other. Intraoperative findings and pathological work-up identified RCC along with adenomatous tissue displaying hemorrhagic pituitary adenoma in one and hemorrhagic RCC in the other. Clinical symptoms of pituitary apoplexy were present in both cases, making pituitary and RCC apoplexy clinically indistinguishable. RCC and concomitant pituitary adenoma are a rare intraoperative finding that must be considered as a differential diagnosis in patients with symptoms of pituitary adenoma apoplexy.

KEYWORDS: Rathke cleft cyst, pituitary adenoma, pituitary apoplexy, anticoagulation

Rathke cleft cysts (RCCs) are benign sellar and suprasellar lesions believed to develop from remnants of the craniopharyngeal duct during embryogenesis. RCCs are usually small and asymptomatic; however, when large, they can present with symptoms related to mass effect on adjacent structures. In rare cases, RCCs have been reported to present with symptoms of pituitary apoplexy—the sudden onset of headache and visual symptoms due to hemorrhagic transformation or infarction of a pituitary lesion. ${ }^{1}$ The coexistence of RCC and pituitary adenoma has been described in up to $2.1 \%$ of patients with sellar lesions. $^{2}$ We report the case of two patients with clinical symptoms of pituitary apoplexy and intraoperative findings of coexisting RCC and nonfunctional pituitary adenoma.

\section{CASE REPORTS}

\section{Case 1}

\section{HISTORY, PRESENTATION, AND EXAMINATION}

A 76-year-old woman experienced acute onset of headache, retro-orbital pain, double vision, and a drooping left eyelid 3 weeks before presentation. She reported that

"These authors have contributed equally to this work.

${ }^{1}$ Department of Neurosurgery, Clinical Neurosciences Center;

${ }^{2}$ Department of Pathology, University of Utah, Salt Lake City, Utah. Address for correspondence and reprint requests: William $\mathrm{T}$. Couldwell, M.D., Ph.D., Department of Neurosurgery, Clinical Neurosciences Center, University of Utah, 175 N. Medical Drive East, Salt Lake City, UT 84132 (e-mail: neuropub@hsc.utah.edu).

Skull Base Rep 2011;1:99-104. Copyright (C) 2011 by Thieme
Medical Publishers, Inc., 333 Seventh Avenue, New York, NY 10001, USA. Tel: +1(212) 584-4662.

Received: December 14, 2010. Accepted after revision: March 22, 2011. Published online: June 9, 2011.

DOI: http://dx.doi.org/10.1055/s-0031-1280737.

ISSN 2157-6971. 
A
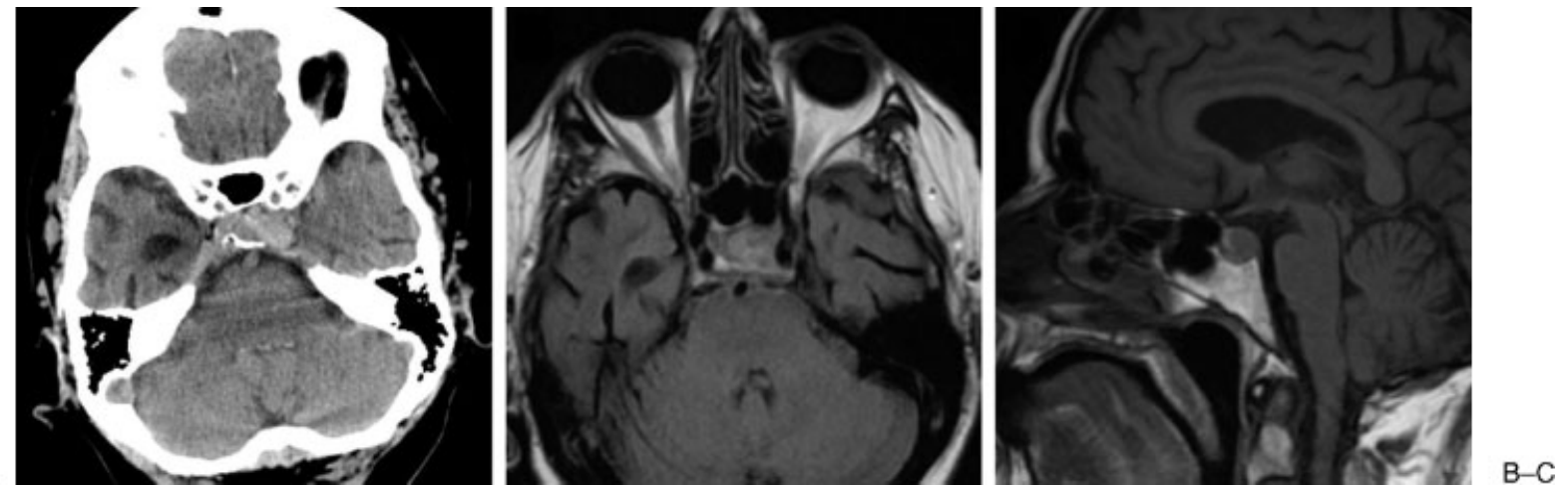

Figure 1 Preoperative imaging of patient 1. (A) Axial CT image obtained preoperatively after onset of headaches showing a mixed hyperdense sellar lesion. T1-weighted axial (B) and sagittal (C) MRI demonstrating a hypointense lesion along the infundibulum consistent with a pituitary adenoma.

her initial symptoms had improved; however, she experienced another event 2 weeks later with worsening headache and double vision. Her medical history was remarkable for a coronary artery bypass graft and aortic valve replacement within a year of presentation; thus, she had been treated with Coumadin (warfarin) and was undergoing anticoagulant therapy during the onset of the symptoms.

Clinical examination of the patient demonstrated a left third nerve palsy and a partial sixth nerve palsy, with limitation of gaze in all directions and significant ptosis. A pituitary endocrinological laboratory work-up was found to be normal, with the exception of a mildly elevated prolactin level of $26.7 \mathrm{ng} / \mathrm{mL}$ (normal $=2.8$ to $20 \mathrm{ng} / \mathrm{mL}$ ).

Magnetic resonance imaging (MRI) demonstrated a left-sided sellar mass displacing the pituitary gland along with the infundibulum consistent with a pituitary adenoma (Fig. 1). A noncontrast head computed tomography (CT) scan obtained after her initial apoplectic episode revealed a sellar hyperdensity consistent with hemorrhage.

\section{OPERATIVE AND HISTOPATHOLOGICAL FINDINGS}

Our diagnosis based on clinical and radiographic data was pituitary adenoma with two episodes of pituitary apoplexy. Following discussion with her cardiothoracic surgeon, the patient's anticoagulation therapy was withheld in the immediate perioperative period. The patient subsequently underwent transsphenoidal resection, at which time the tissue extracted was notable for adenoma and mucoid (RCC) tissues. Intraoperatively, the sella turcica was found to be eroded, and, when the dura mater was opened, a hemorrhagic pituitary mass was expressed. Additionally, a mucinous lesion was resected, consistent with RCC. Pathological analysis confirmed the diagnosis of both nonfunctional hemorrhagic pituitary adenoma and RCC-type epithelia and content conclusive for RCC (Fig. 2).
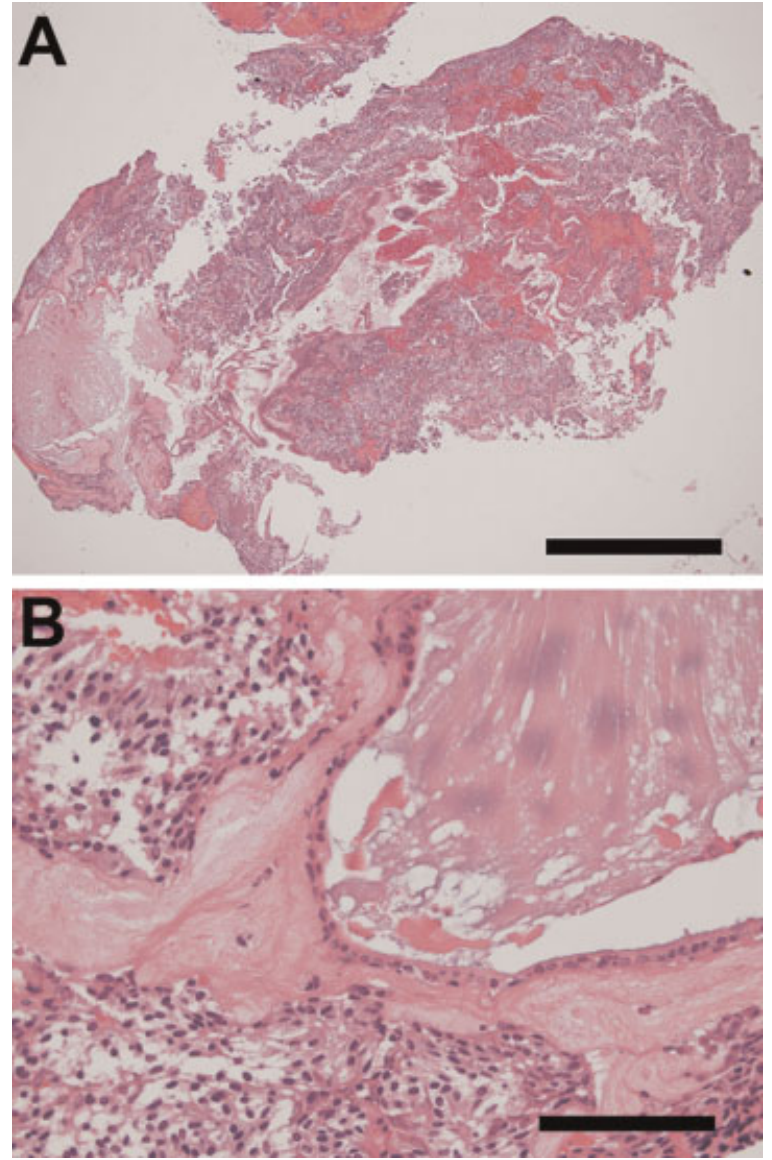

Figure 2 Histopathology of tissue from patient 1. (A) Lowmagnification view showing a cystic, epithelium-lined lesion filled with mucinous material (left to center) surrounded by cellular and hemorrhagic tissue. (B) High-magnification image showing the interface of the RCC (upper right) and the adjacent pituitary adenoma. Formalin-fixed, paraffin-processed sections stained with hematoxylin and eosin. Scale bar: $1 \mathrm{~mm}(\mathrm{~A})$ and $0.1 \mathrm{~mm}(\mathrm{~B})$ 


\section{POSTOPERATIVE COURSE}

At 5-month follow-up examination, the patient was free of complaints and neurologically intact; pituitary-related hormone levels were within normal limits. MRI showed complete resection of the pituitary tumor.

\section{Case 2}

\section{HISTORY, PRESENTATION, AND EXAMINATION}

A 67-year-old man reported an acute onset of headaches 6 months before presentation. He described the headaches to be constant and at the same level since onset. His medical history was significant for atrial fibrillation, for which he was receiving anticoagulation therapy with Coumadin (warfarin). His clinical examination was free of any focal neurological deficits.

Endocrinological laboratory findings were consistent with a diagnosis of nonsecreting adenoma. Preoperative MRI (Fig. 3) displayed an $18 \times 22.5-\mathrm{mm}$ sellar mass in contact with the optic chiasm that appeared to be an RCC, because the images showed an internal hypointense cystic lesion on T1-weighted MRI with gadolinium enhancement.

\section{OPERATIVE AND HISTOPATHOLOGICAL FINDINGS}

On the basis of the patient's clinical presentation and MRI studies, the operation was performed via a transsphenoidal approach. After removal of the sellar floor bone and opening of the dura, a hemorrhagic mucoid material bulged through the incision. The mass was removed in total for pathological analysis. After removal of the specimen, a second mass identified as a pituitary adenoma was visible; it was subsequently removed with curettage. Histopathological analysis described a nonsecreting pituitary adenoma and large amounts of colloid and mucoid material with simple ciliated and mucinous columnar epithelium consistent with RCC (Fig. 4).

\section{POSTOPERATIVE COURSE}

The patient was readmitted on postoperative day 7 for recurrent epistaxis; he was successfully treated with nasal packing and was discharged 3 days later. The patient's anticoagulation therapy, which had been stopped before surgery, was resumed upon discharge from the hospital. At the 1-month follow-up examination, results of the patient's neurological examination were normal. He did not complain about constant headaches, and his pituitary-related hormone levels were within normal limits.

\section{DISCUSSION}

RCCs have a wide spectrum of clinical manifestations and imaging features. ${ }^{3-6}$ Although many cases of RCC are asymptomatic, if the cyst becomes large enough, patients can present with symptoms from compression of the adjacent structures including headache, visual deficits, and pituitary dysfunction. ${ }^{7}$ It is rare for an RCC to present with pituitary apoplexy. Because RCCs have varied T1- and T2-weighted MRI qualities, they may be indistinguishable from pituitary hemorrhage on MRI. In a recent series of six patients with RCC presenting with apoplexy, all patients had preoperative MRI consistent with hemorrhagic pituitary tumor; however, only two patients were found to have hemorrhage at the time of surgery. ${ }^{1}$

RCCs rarely coexist with pituitary adenoma. ${ }^{2,8} \mathrm{~A}$ review of 374 patients who underwent surgery for sellar or juxtasellar lesions found concomitant RCC and pituitary adenoma in 8 patients $(2.1 \%)$, all of whom presented with symptoms from functional growth hormone or adrenocorticotrophic hormone-producing adenomas. ${ }^{2}$ Coexisting RCC and nonfunctional pituitary adenomas are even more rare. ${ }^{5}$

Our first case is unique in that the patient had both RCC and a nonfunctional pituitary adenoma and presented with pituitary apoplexy. As in other reports, preoperative imaging favored a diagnosis of hemorrhagic
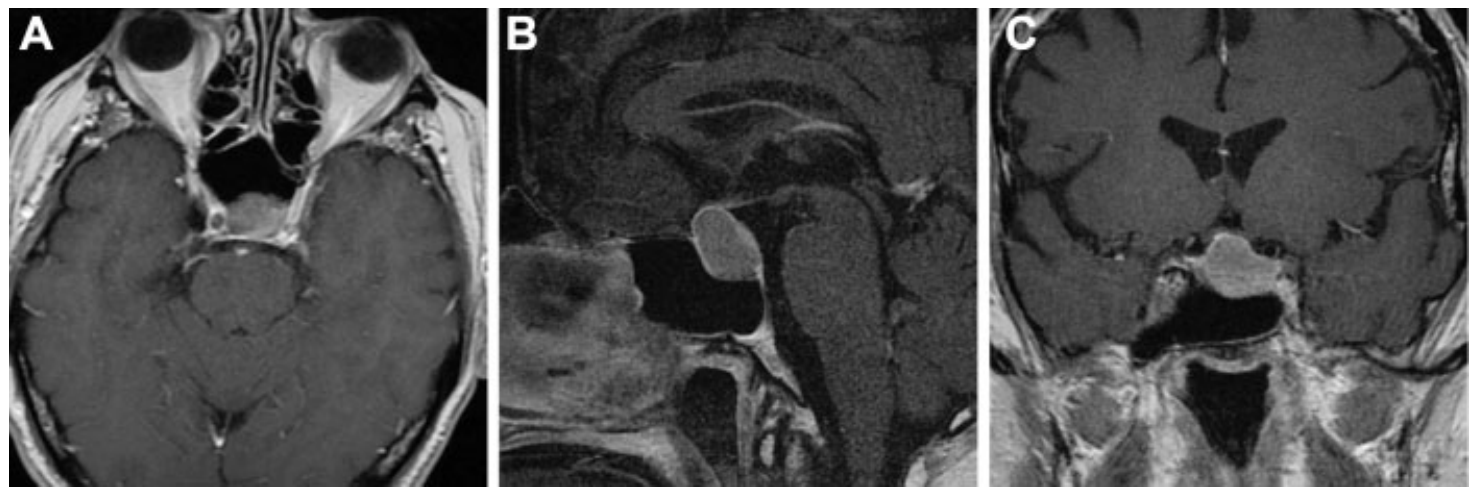

Figure 3 Preoperative imaging of patient 2. Preoperative axial (A), sagittal (B), and coronal (C) T1-weighted MRI with gadolinium enhancement displaying a sellar mass in contact with the optic chiasm, consistent with an RCC. The image shows an internal hypointense cystic lesion. 


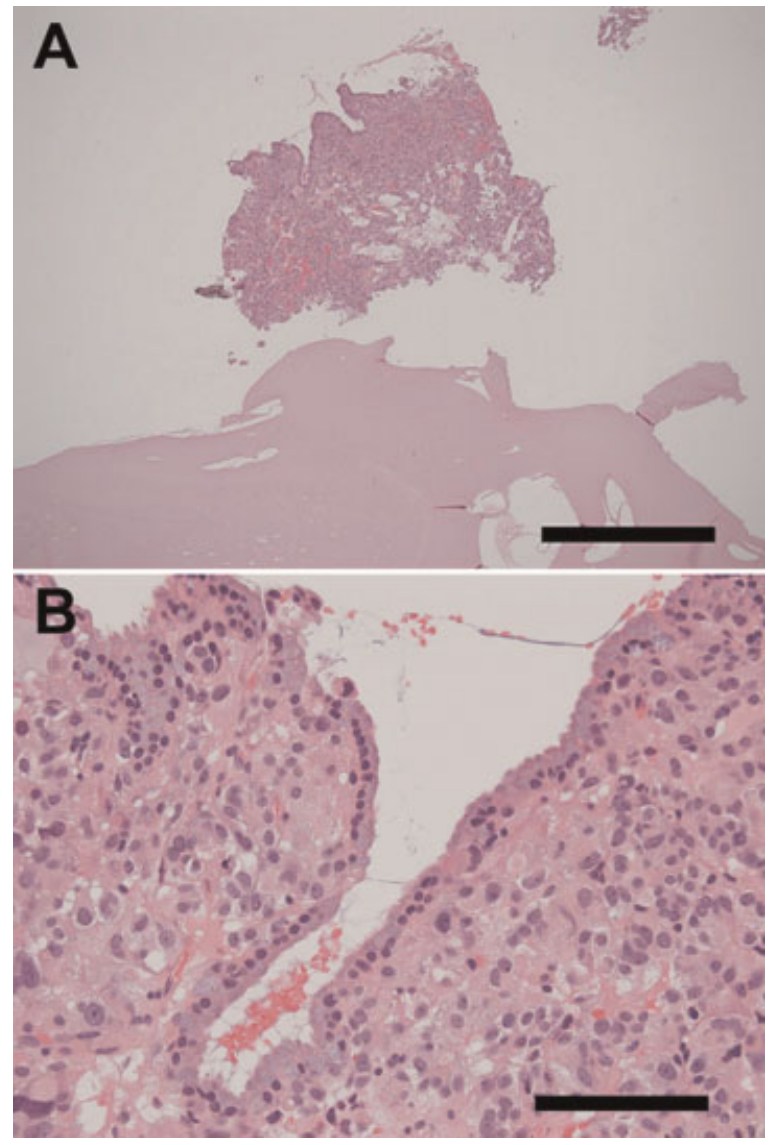

Figure 4 Histopathology of tissue from patient 2. (A) Lowmagnification view showing a fragment of cellular tissue including a portion of the epithelial lining of the RCC and adjacent pituitary adenoma (top) and a small portion of the voluminous mucoid cyst contents (bottom). (B) High-magnification view of the simple ciliated and mucinous columnar epithelium of the RCC surrounded by pituitary adenoma. Formalin-fixed, paraffin-processed sections stained with hematoxylin and eosin. Scale bar: $1 \mathrm{~mm}(\mathrm{~A})$ and $0.1 \mathrm{~mm}(\mathrm{~B})$.

pituitary tumor, and the presence of the RCC was only discovered at the time of surgery. The imaging of the second case indicated a cystic component favoring an $\mathrm{RCC}$ and the diagnosis of hemorrhagic RCC was discovered during surgery.

Of particular interest is that both individuals were taking Coumadin (warfarin) for anticoagulation at the time of symptom onset. Although the patients' International Normalized Ratio (INR) was not measured at the time of symptom onset, both patients were compliant with their medication regime and thus the INR values were presumably in the therapeutic range. Anticoagulation therapy may lead to spontaneous pituitary hemorrhage in patients with pituitary adenomas as reported in three cases. ${ }^{9-11}$ Pituitary adenomas have an increased risk of ischemic apoplexy as they compress supplying vessels or outgrow effective blood supply. ${ }^{12,13}$ Because of vascular pathology, hemorrhagic pituitary adenomas can be observed in up to $25 \%$ of patients suffering from pituitary adenoma. ${ }^{14}$ It is possible that anticoagulation therapy increases the likelihood of having a symptomatic hemorrhagic event as our patients experienced.

Only a few cases of RCC and concomitant pituitary adenoma have been described. Symptomatic RCC may present by compression of surrounding structures or by apoplexy of the pituitary gland. Presentation may also occur with apoplexy of the RCC as our cases demonstrate. Anticoagulation therapy precipitates pituitary adenomas and possibly RCCs to hemorrhage and may increase the risk of symptomatic apoplexy. Our cases demonstrate the importance of patient education and physician's alertness in patients with acute onset of headache and history of pituitary adenoma or RCC.

\section{ACKNOWLEDGMENT}

The authors thank Kristin Kraus, M.Sc., for excellent editorial assistance in preparing this paper.

\section{REFERENCES}

1. Binning MJ, Liu JK, Gannon J, Osborn AG, Couldwell WT. Hemorrhagic and nonhemorrhagic Rathke cleft cysts mimicking pituitary apoplexy. J Neurosurg 2008;108(1):3-8

2. Sumida M, Migita K, Tominaga A, Iida K, Kurisu K. Concomitant pituitary adenoma and Rathke's cleft cyst. Neuroradiology 2001;43(9):755-759

3. Brassier G, Morandi X, Tayiar E, et al. Rathke's cleft cysts: surgical-MRI correlation in 16 symptomatic cases. J Neuroradiol 1999;26(3):162-171

4. Sade B, Albrecht S, Assimakopoulos P, Vézina JL, Mohr G. Management of Rathke's cleft cysts. Surg Neurol 2005;63(5): 459-466, discussion 466

5. Vancura RW, Jacob KM, Damjanov I. A 70-year-old man with diplopia, nausea, and vomiting. Rathke cleft cyst concomitant with pituitary adenoma. Arch Pathol Lab Med 2006;130(3):403-404

6. Kucharczyk W, Peck WW, Kelly WM, Norman D, Newton TH. Rathke cleft cysts: CT, MR imaging, and pathologic features. Radiology 1987;165(2):491-495

7. Nishioka H, Haraoka J, Izawa H, Ikeda Y. Magnetic resonance imaging, clinical manifestations, and management of Rathke's cleft cyst. Clin Endocrinol (Oxf) 2006;64(2):184-188

8. Noh SJ, Ahn JY, Lee KS, Kim SH. Pituitary adenoma and concomitant Rathke's cleft cyst. Acta Neurochir (Wien) 2007; 149(12):1223-1228

9. Nagarajan DV, Bird D, Papouchado M. Pituitary apoplexy following anticoagulation for acute coronary syndrome. Heart 2003;89(1):10

10. Tan TM, Caputo C, Mehta A, Hatfield EC, Martin NM, Meeran K. Pituitary macroadenomas: are combination antiplatelet and anticoagulant therapy contraindicated? A case report. J Med Case Reports 2007;1:74

11. Nourizadeh AR, Pitts FW. Hemorrhage into pituitary adenoma during anticoagulant therapy. JAMA 1965;193: 623-625 
12. Cardoso ER, Peterson EW. Pituitary apoplexy: a review. Neurosurgery 1984;14(3):363-373

13. Semple PL, Webb MK, de Villiers JC, Laws ER Jr. Pituitary apoplexy. Neurosurgery 2005;56(1):65-72, discussion 72-73
14. Lubina A, Olchovsky D, Berezin M, Ram Z, Hadani M, Shimon I. Management of pituitary apoplexy: clinical experience with 40 patients. Acta Neurochir (Wien) 2005; 147(2):151-157, discussion 157 